\title{
Macrococcus caseolyticus
}

National Cancer Institute

\section{Source}

National Cancer Institute. Macrococcus caseolyticus. NCI Thesaurus. Code C86506.

A species of anaerobic, Gram positive, large cocci shaped bacteria assigned to the

phylum Firmicutes. This species is nonmotile, nonhemolytic, produces acid from maltose, beta $D$ fructose and $D$ trehalose, reduces nitrate, produces acetoin, catalase and oxidase positive and urease negative. M. caseolyticus is a nonpathogenic, commensal organism of the skin and is commonly isolated from foods such as meat and cheese. 